\title{
Evaluation of Aircraft Cabin Air Quality Considering Breath by People on Board*
}

\author{
Hidefumi SAITO** and Takeo KAJISHIMA*** \\ ** R\&D Department, Aircraft Equipment Div. Shimadzu Corporation \\ 1 Nishinokyo-Kuwabara-Cho, Nakagyo-Ku, Kyoto, 604-8511, Japan \\ E-mail: h-saito@shimadzu.co.jp \\ *** Department of Mechanical Engineering, Osaka University \\ 2-1 Yamadaoka, Suita, Osaka, 565-0871, Japan
}

\begin{abstract}
For the application to optimal design and effective operation of air-conditioning system in an aircraft cabin, we propose an indicator to represent the air quality. It was derived from the result of the large-eddy simulation (LES) of turbulent flow with the immersed boundary (IB) method, which took into account the respiration of passengers. The cabin air quality is defined as an oxygen concentration of inhalation air (OCIA) per each breath. Degree of air renewal in reference volume is a dominant factor of air quality, OCIA. Intensity of time-averaged velocities by cross-sectional components closely correlates with the degree of the air replacement, and becomes the most suitable indicator of air quality. In case of decrease of the intensity, each of two different flow conditions, namely stagnation and circular flow just around a mouth, was found out at the reference volume as inactivation elements of the air renewal.
\end{abstract}

Key words: Air Quality, Inhalation Air, Unsteady Flow, Large-Eddy Simulation, Immersed Boundary Method, Oxygen Concentration

\section{Introduction}

In accordance with the recent research progress about impact of substances in air on human body ${ }^{(1)(2)}$, the regulations for cabin air ${ }^{(3)(4)}$ have been continually updated.

A consideration of the priority in a particular sub-area of room may enable more efficient operation of air conditioning than a usual operation based on the standard ${ }^{(5)}$ treating whole room uniformly. To shape this idea, personalized ventilation (PV) has been introduced by Fanger ${ }^{(6)}$ to architectural engineering. Based on the concept of PV, he supplied fresh air directly and gently into breathing zone, and evaluated its effect. Melikov et al. ${ }^{(7)}$ presented many types of air terminal device (ATD) as personal outlet for OA worker. They evaluated performances of ATDs for temperature control and reducing contaminations in inhalation air. Gao et al. ${ }^{(8)}$ simulated flow field of a whole room that includes a typical ATD and a computational thermal manikin (CTM). Zitek et al. ${ }^{(9)}$ installed ATD at a back surface of anterior seat as a PV system for a passenger in aircraft, and evaluated its effectiveness both experimentally and computationally. There are some studies focused on transient conditions of air in a room to design comfortable space for an occupant. Hiyama et al. ${ }^{(10)}$ calculated transient temperature field by Response Factor Method by which heat convection was treated as heat pulses in a steady flow field. In the method, the $k-\varepsilon$ turbulent model was applied to making the steady flows in advance for the calculation of heat convection. Zhu et al. ${ }^{(11)}$ studied transient respiration flow near a mouth to investigate 
movements of pollutant substances.

Incidentally, a gasper ${ }^{(1)}$, i.e. outlet above each passenger seat and a kind of PV, has been popular as a tool of air conditioning system in vehicles since the time when an airliner service was started. We suggested that the gasper would be exploited more widely than conventional use like a temporary cooler, and would become a suitable tool for improving passenger's air quality by using oxygen and moisture enriched air ${ }^{(12)}$. For fulfillment of the suggestion, it is important to evaluate passenger's air quality precisely. In the computational studies about $\mathrm{PV}^{(8)(10)(11)}$, they use the $k-\varepsilon$ model to calculate respiration flow or ventilation flow in the steady upward wind that simulated a thermal plume. Their results showed that the flow field was steady, or that fluctuation of flow by respiration was steadily cyclic. However, jet of supplied air and thermal plume in real field are not steady and are easily disturbed by various conditions. Exhaled air from an occupant often remains around his or her mouth. Correspondingly to inactivation of air renewal, a part of the exhaled air is inhaled again in next inhaling action. Consequently, the respiration flow is not steadily cyclic.

Recently, large-eddy simulation (LES) prevails for calculation of unsteady flow field and is applied even to complex and actual flow field. In our previous study ${ }^{(13)}$, we tried to apply LES to our cabin model. LES, instead of using the $k-\varepsilon$ model, can essentially calculate unsteady flow field involving transient respiration flow of each passenger. Furthermore, the immersed boundary (IB) method was also used in our previous study. IB method is useful to simplify the calculation including complicated configuration of solid parts such as human bodies, seats, and cabin interiors. Our previous study showed that a deterioration of air quality occurred in some conditions.

In this study, we progressed in elucidating inhalant air quality and extracting dominant factor of the air quality. Flow data averaged during the period of one inhaling or exhaling action is calculated for each passenger. Then, from a viewpoint of the design of air-conditioning, we investigate flow parameters that indicate states of the air quality. This paper shows our study about the air quality indicators focused on passenger's respiration, and usefulness of the most suitable indicator that we select in consideration for the flow field.

\section{Computational Model and Governing Equations}

\subsection{Model of Cabin}

As shown in Fig. 1, a computational model is designed based on cabin of Boeing 767 aircraft, of which dimensions are shown in the reference ${ }^{(15)}$. A half of one row is selected for the computational domain and flow field is assumed to be symmetry about the centerline. The computational domain is bounded by $0 \leq x \leq 2,420 \mathrm{~mm}, 0 \leq y \leq 2,120 \mathrm{~mm}$, $0 \leq z \leq 800 \mathrm{~mm}$. We assumed the flow field is periodic in the z-direction corresponding to the pitch of the seat as shown in Fig. 1(b). The average stream rate in the z-direction is given corresponding to the prescribed amount of ventilation air ${ }^{(14)}$. Air that is supplied from ventilation system into the cabin is in the part of the ceiling, and the same amount of air is extracted through the part of the floor from the cabin. Inhaling or exhaling air flow is set at each mouth position of the human body.

The computational domain is divided into $121 \times 106 \times 40$ uniform cubic cell of $20 \mathrm{~mm}$ per side. Zhu, et al. ${ }^{(16)}$ demonstrated that exhalation jet width was approximately $20 \mathrm{~mm}$ by using the Particle Image Velocimetry technique and a thermal manikin. Therefore, the grid size of our model makes it possible to observe the interaction between respiration flow and other flows of whole domain essentially. Furthermore, even though using such coarse grid, kinetic energy ratio of sub-grid scale to grid scale is not more than 0.5 in the whole analytical domain $^{(13)}$. 
Cabin interiors (i.e. ceilings, sidewall, seats, and floor) and human bodies are represented by a particular method ${ }^{(17)}$ in IB method ${ }^{(18)}$. At the cell including both solid part and fluid part (air part), the rate of solid occupation ("solid rate") is given, and the flow velocity at the cell is forced according to the solid rate.

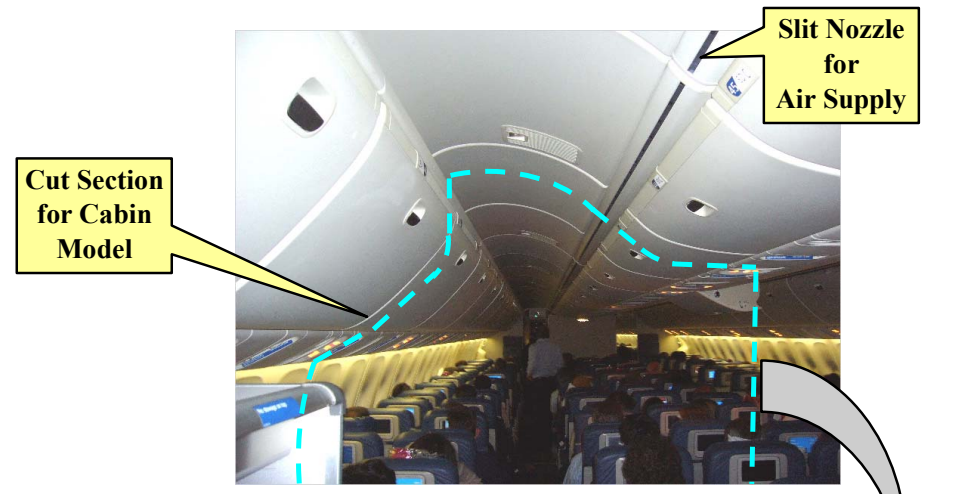

(a) Actual cabin space of a B767 aircraft

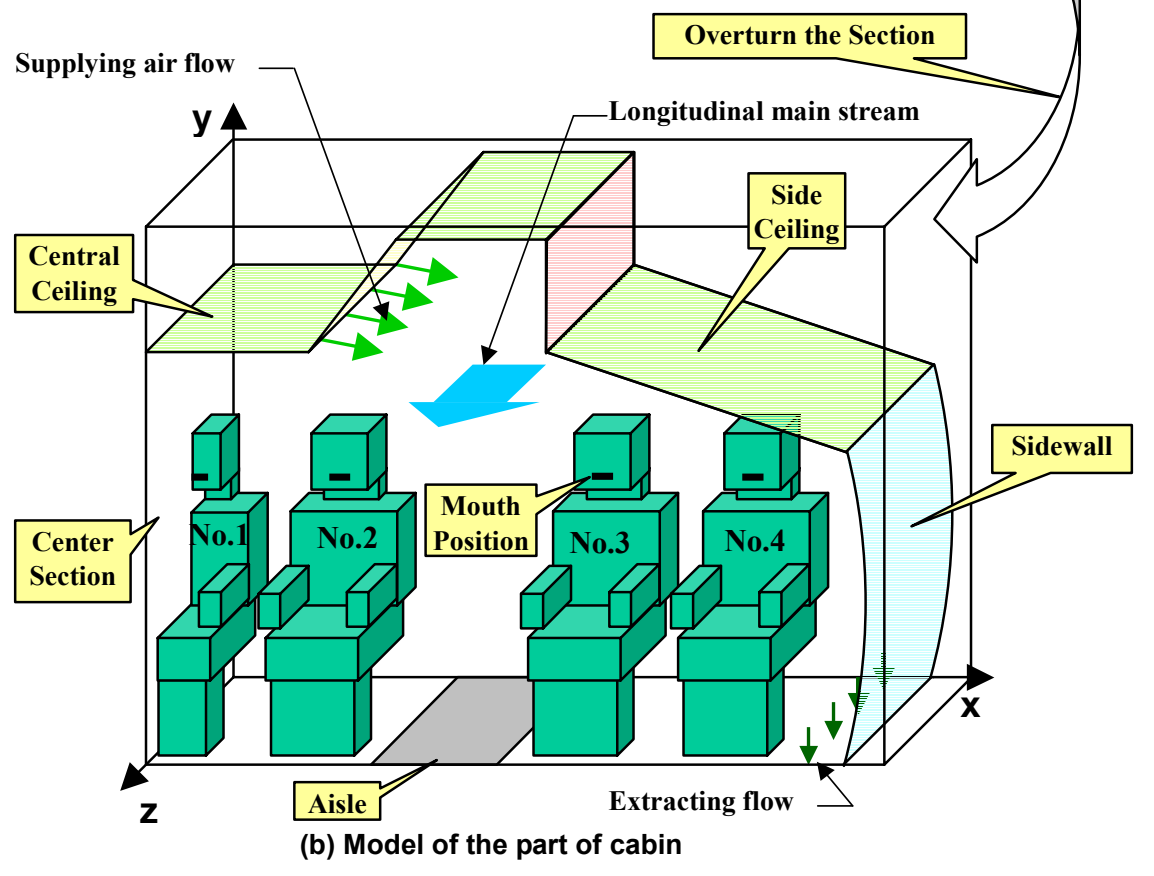

Fig.1 Computational domain in the cabin

\subsection{Governing Equations}

In LES, a filter separates the Grid Scale (GS) field from Sub-Grid Scale (SGS) turbulence. The governing equations for filtered flow field are the followings.

$$
\begin{aligned}
& \nabla \cdot \mathbf{u}=0, \\
& \frac{\partial \mathbf{u}}{\partial t}=-\nabla \cdot(\mathbf{u u})-\frac{1}{\rho} \nabla P+\nabla \cdot(2 v \mathbf{D})+\mathbf{f}, \\
& \frac{\partial \theta}{\partial t}=-\nabla \cdot(\theta \mathbf{u})+\nabla \cdot(\alpha \nabla \theta), \\
& \frac{\partial \xi}{\partial t}=-\nabla \cdot(\xi \mathbf{u})+\nabla \cdot(D \nabla \xi),
\end{aligned}
$$


where $\mathbf{u}$ is the velocity vector $(\mathrm{m} / \mathrm{s}), \mathbf{D}$ the deformation rate tensor $\left(\mathrm{s}^{-1}\right), P$ the pressure $(\mathrm{Pa}), \theta$ the temperature $(\mathrm{K}), \xi$ the oxygen concentration, $\rho$ the density $\left(\mathrm{kg} / \mathrm{m}^{3}\right), \quad v$ the kinematic viscosity of air $\left(\mathrm{m}^{2} / \mathrm{s}\right), \alpha$ the temperature diffusivity of air $\left(\mathrm{m}^{2} / \mathrm{s}\right), D$ the oxygen molecule diffusion coefficient into nitrogen molecule $\left(\mathrm{m}^{2} / \mathrm{s}\right)$.

The body force $\mathbf{f}$ consists of buoyancy (in y-direction) and driving force of main stream (in z-direction). Since the variation of fluid density due to change of composition is not more than $0.7 \%$, we do not take into account buoyancy by oxygen concentration. Hence, the buoyancy is treated by Boussinesq approximation

$$
f_{y}=g \beta\left(\theta-\theta_{0}\right)=\frac{g \Delta \theta}{\theta_{0}},
$$

where $\Delta \theta\left(=\theta-\theta_{0}\right)$ is the temperature difference, $\theta_{0}(=298 K)$ the standard ambient temperature, $g\left(=9.81 \mathrm{~m} / \mathrm{s}^{2}\right)$ the acceleration of gravity, $\beta$ the coefficient of volume expansion. The coefficient $\beta$ is defined as $-(1 / \rho)|\partial \rho / \partial \theta|_{P}$ and becomes $1 / \theta_{0}$ in case where air is assumed to be ideal gas and $|\Delta \theta| \ll \theta_{0}$. The driving force of the main stream is given by

$$
f_{z}=\frac{\Delta P}{\rho},
$$

where $\Delta P\left(=-8.0 \times 10^{-4} \mathrm{~Pa} / \mathrm{m}\right)$ is the pressure gradient.

\subsection{Sub-Grid Scale Model}

The kinematic viscosity in Eq.(2) is given by

$$
v=v_{0}+v_{S G S},
$$

where the subfix " 0 " indicates the molecular diffusivity part and the subfix "SGS" denotes the SGS eddy diffusivity part. $v_{S G S}$ is defined as the Smagorinsky model and given by

$$
v_{S G S}=\left(C_{s} f_{s} \Delta\right)^{2}|\mathbf{D}|
$$

where $\Delta\left(=\sqrt[3]{\Delta_{x} \Delta_{y} \Delta_{z}}\right)$ is the filter width $\left(\Delta_{x}: \mathrm{x}\right.$-grid size, $\Delta_{y}:$ y-grid size, $\Delta_{z}:$ z-grid size), $C_{S}$ the Smagorinsky coefficient $(=0.10), f_{S}$ the damping function of van Driest type, $|\mathbf{D}|$ the intensity of deformation rate tensor. The damping function $f_{S}$ is given by

$$
f_{s}=1-\exp \left(-\frac{l u_{\tau} / v_{0}}{25}\right) .
$$

To simplify $f_{S}$, we selected an averaged wall-friction velocity $u_{\tau}$ for the whole computational domain. The distance between the referring point and the nearest solid part is denoted by $l$.

SGS eddy diffusivity, $\alpha$ in Eq.(3), and $D$ in Eq.(4) are given by

$$
\begin{gathered}
\alpha=\alpha_{0}+\frac{v_{S G S}}{P r_{S G S}}, \\
D=D_{0}+\frac{v_{S G S}}{S c_{S G S}},
\end{gathered}
$$


where SGS turbulent Prandtl number $P r_{S G S}$ and SGS turbulent Schmidt number $S c_{S G S}$ are 0.5 . They are the same as in LES for thermal convection ${ }^{(19)}$ based on previous study ${ }^{(20)}$.

Values for the molecular diffusivity part are given as $v_{0}=1.57 \times 10^{-5} \mathrm{~m}^{2} / \mathrm{s}$ in Eqs.(7) and (9), $\alpha_{0}=2.18 \times 10^{-5} \mathrm{~m}^{2} / \mathrm{s}$ in Eq.(10), $D_{0}=1.80 \times 10^{-5} \mathrm{~m}^{2} / \mathrm{s}$ in Eq.(11).

\subsection{Calculation Schemes}

The governing equations are spatially discretized based on the staggered arrangement, in which scalars (pressure, temperature, and oxygen concentration) are on the center of each cell. The second-order central finite-difference method is applied.

We adopt Simplified Maker and Cell (SMAC) method for the velocity-pressure coupling. For the prediction step, the second-order Adams-Bashforth scheme is applied. The successive over-relaxation (SOR) method is used for the Poisson equation of the pressure. The time-marching for the temperature and oxygen concentration fields are also calculated by the second-order Adams-Bashforth scheme.

The IB method of body-force type ${ }^{(17)}$ was used in our simulation.
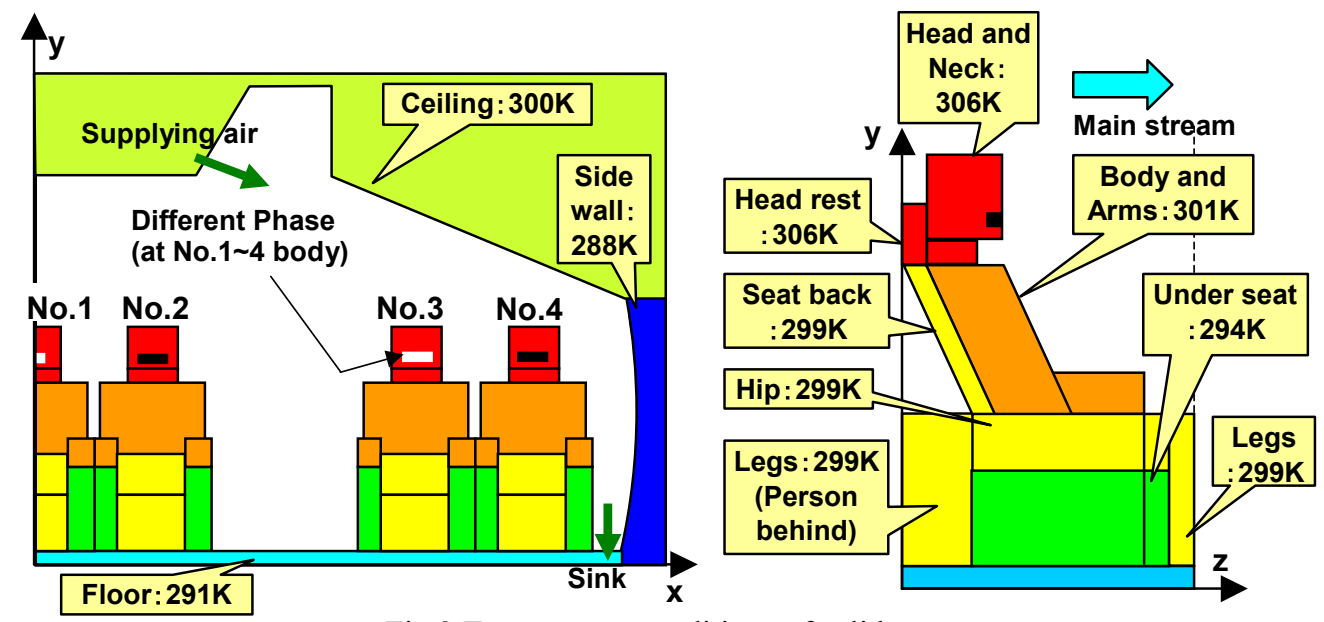

Fig.2 Temperature conditions of solid parts

\subsection{Boundary Conditions}

Boundary condition of center section $(x=0 \mathrm{~mm})$ is mirror boundary with zero normal velocity, and boundary conditions for z-direction $(z=0 \mathrm{~mm}$ and $z=800 \mathrm{~mm})$ are periodic boundaries as described in the section 2.1 .

Boundary conditions at the solid surfaces are no-slip and no-permeable. As shown in Fig.2, fixed temperatures of the cells filled with solid are given. To deal with non-permeability of oxygen at the solid surface, mass-flux values of both the advection term and the diffusion term in Eq.(4) are forced to zero at the boundary cells.

Table 1 Conditions of the supplied air from the ceiling

\begin{tabular}{|c|c|c|c|}
\hline Condition & Velocity & Temperature range & Oxygen concentration \\
\hline A & $0.25 \mathrm{~m} / \mathrm{s}$ & $292 \sim 297 \mathrm{~K}$ & 0.21348 \\
\hline $\mathrm{B}$ & $0.50 \mathrm{~m} / \mathrm{s}$ & $292 \sim 297 \mathrm{~K}$ & 0.21149 \\
\hline $\mathrm{C}$ & $0.25 \mathrm{~m} / \mathrm{s}$ & $287 \sim 292 \mathrm{~K}$ & 0.21348 \\
\hline $\mathrm{D}$ & $0.50 \mathrm{~m} / \mathrm{s}$ & $287 \sim 292 \mathrm{~K}$ & 0.21149 \\
\hline $\mathrm{E}$ & $0.25 \mathrm{~m} / \mathrm{s}$ & $287 \sim 297 \mathrm{~K}$ & 0.21348 \\
\hline $\mathrm{F}$ & $0.50 \mathrm{~m} / \mathrm{s}$ & $287 \sim 297 \mathrm{~K}$ & 0.21149 \\
\hline
\end{tabular}


In this study, 6 conditions are set as shown in Table 1. The temperature of the supplied air is a little lower than that of cabin space, and fluctuates by $0.425 \mathrm{cycles} / \mathrm{min}$. Though the intensity of fluctuation is higher than that practically supposed, it is intended to clarify the relationship between OCIA and the indicators. Condition-A is basic condition of the supplied air. Condition-B, -D, and -F are higher velocity conditions. Condition-C and -D are lower supply air temperature conditions. Condition-E and $-\mathrm{F}$ are wider temperature amplitude conditions. Oxygen concentration of the supplied air is decided based on total amount of supplementary oxygen.

As a boundary condition at the mouth, periodic inflow and outflow are given considering breath of passengers. Flow velocity by respiration at mouth position is given by sinusoidal pattern with the frequency of 17 cycles per minute. Since a person is breathing in different phase from another person generally, the respiration phases at four bodies are different each other in multiple numbers of 90 degrees. Total volume of one inhaling action of one person is $400 \mathrm{cc}^{(21)}$. Conditions of exhalation air are set as fixed values, namely $\theta=310 \mathrm{~K}$ and $\xi=0.1627^{(21)}$.

\section{Reference Volume and Indicator of Air Quality}

The air quality is evaluated by an oxygen concentration of inhalation air (OCIA), which is calculated by operation of time-averaging during a period of one inhalation.

As a reference volume, a space in front of mouth (SIFM) is defined to find a flow parameter that correlates to the air quality. Figure 3 depicts the SIFM. The size of the SIFM is approximately $128.4 \mathrm{~mm}(x) \times 108.4 \mathrm{~mm}(y) \times 44.2 \mathrm{~mm}(z)$, that corresponds to the volume of

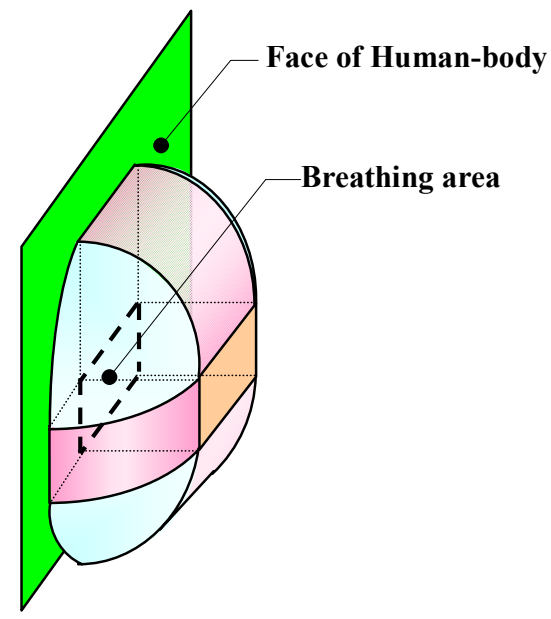

Fig.3 Shape of the SIFM inhalation air of one breath.

For the purpose of calculation of indicators of air quality, following equations are defined for a certain quantity $f$. Time averaged value $\bar{f}$ is given by

$$
\bar{f}=\frac{1}{T_{i}} \int_{t}^{t+T_{i}} f d t,
$$

where $T_{i}$ is time of one inhalation period. Spatially averaged inside SIFM $\langle f\rangle$ is given by

$$
\langle f\rangle=\frac{1}{V_{\Omega}} \int_{V_{\Omega}} f d V,
$$

where $V_{\Omega}$ is volume of the SIFM. Standard deviations $\sigma_{f}$ is given by

$$
\sigma_{f}=\sqrt{\left\langle\overline{f^{2}}\right\rangle-\langle\bar{f}\rangle^{2}}
$$

We select four indicators. As a representative of velocity vector, "intensity of time-averaged velocities" $\bar{V}_{3 D}$ is defined by

$$
\bar{V}_{3 D}=\sqrt{\langle\bar{u}\rangle^{2}+\langle\bar{v}\rangle^{2}+\langle\bar{w}\rangle^{2}}
$$

where $\mathrm{u}, \mathrm{v}$ and $\mathrm{w}$ are $\mathrm{x}, \mathrm{y}$ and $\mathrm{z}$-component of velocities. In case where stable stream in the SIFM during one inhaling period primarily affects air renewal, $\bar{V}_{3 D}$ correlates closely with 
the OCIA. In addition, “intensity of time-averaged 2D velocities" $\bar{V}_{2 D}$ is defined by

$$
\bar{V}_{2 D}=\sqrt{\langle\bar{u}\rangle^{2}+\langle\bar{v}\rangle^{2}}
$$

It is tested because the normal component $\mathrm{w}$ is thought to be less influential in comparison with other components.

To represent degree of fluctuation of the velocity vector, "intensity of velocity deviations" $\bar{S}_{3 D}$ is defined by

$$
\bar{S}_{3 D}=\sqrt{\sigma_{u}^{2}+\sigma_{v}^{2}+\sigma_{w}^{2}},
$$

where $\sigma_{u}, \sigma_{v}$ and $\sigma_{w}$ are standard deviations of $\mathrm{u}, \mathrm{v}$ and $\mathrm{w}$ respectively during a period of one inhalation. If fluctuation component of flow in the SIFM during one inhalation primarily affects air renewal, $\bar{S}_{3 D}$ should correlate closely with the OCIA.

A combined indicator,

$$
\overline{V S}_{3 D}=\bar{V}_{3 D}+\bar{S}_{3 D} \text {, }
$$

is also tested in our study.

\section{Results and Discussion}

\subsection{Correlation between Indicators and OCIA}

Figures $4 \sim 7$ show some typical correlations between four indicators $\left(\bar{V}_{2 D}, \bar{V}_{3 D}, \bar{S}_{3 D}\right.$, and $\overline{V S}_{3 D}$ ) and OCIA.

Figure 4 shows the result of No.2-body in Condition-A as an example with wide range OCIA fluctuations. The two indicators, namely $\bar{V}_{3 D}$ and $\bar{V}_{2 D}$, are correlated with OCIA. From comparison between $\bar{V}_{3 D}$ and $\bar{V}_{2 D}$, dots of data by these two indicators form into almost the same distribution pattern. Nevertheless $\bar{V}_{2 D}$ exists in a little left area (lower value of indicator), and slant of the $\bar{V}_{2 D}$ distribution is stronger than that of $\bar{V}_{3 D}$. Incidentally, $\bar{S}_{3 D}$ does not fluctuate in accordance with the OCIA. Correlation between $\overline{V S}_{3 D}$ and the OCIA is weaker than that between $\bar{V}_{3 D}$ and the OCIA, due to an effect of $\bar{S}_{3 D}$.

Figure 5 shows the pattern of No.4-body in Condition-A as an example with narrow range OCIA fluctuations. The plots of OCIA of No.4-body are kept around 0.209 even though the temperature of the supplied air fluctuates. In this case, Correlation between $\bar{V}_{3 D}$ or $\bar{V}_{2 D}$ and the OCIA is not clear, and the pattern is different from that in Fig.4.

Figure 6 shows the data pattern of No.2-body in Condition-B of which velocity of the supplied air is increased from $0.25 \mathrm{~m} / \mathrm{s}$ to $0.50 \mathrm{~m} / \mathrm{s}$. From comparison between Fig. 4 and Fig.6, low OCIA data and normal OCIA data tend to be separated, nevertheless, different properties of correlations between the indicators and the OCIA are not recognized.

Figure 7 shows the different OCIA pattern in the case of No.2-body in Condition-D of which temperature range of the supplied air is set 5-degree lower than Condition-B. The most of OCIA data do not exist in the vicinity of 0.209 , where the data without deterioration concentrate as shown in Fig.5.

Correlation coefficients of the four indicators with the OCIA are summarized in Fig.8. In the charts, the ranges of OCIA ( $\triangle O C I A)$, as shown in Fig.4 for example, are also indicated. In case of small $\triangle O C I A$, the correlation coefficient has less meaning of a response of the indicators to the OCIA because the smaller variance makes the correlation coefficient larger. Therefore, it is important to focus on the correlation coefficients calculated by the data in the cases of large $\triangle O C I A$. From comparison of $\triangle O C I A$ of all results in Fig.8, $\triangle O C I A$ of No.2-body is greater than 0.005 for all calculated conditions. It can be also recognized that air quality at No.2-body is liable to deteriorate. It is also suggested that air quality at No.4-body position is kept good because $\triangle O C I A$ of 
No.4-body is smaller than 0.0025 for all calculated conditions.

In Fig.8, the correlation coefficient between $\bar{V}_{2 D}$ and the OCIA (and also between $\bar{V}_{3 D}$ and the OCIA) becomes approximately 0.7 or higher at No.2-body except in Condition-C and Condition-D. Those in Condition-C and Condition-D are $0.5 \sim 0.6$ as a little lower values than those in the other conditions, since the data pattern is unevenly distributed as shown in Fig.7, that indicates few OCIA points within the region of $0.208 \sim 0.210$ where OCIA data normally exist. Ordinarily, the correlation coefficients of $\bar{V}_{2 D}$ with the OCIA are a little higher than those of $\bar{V}_{3 D}$. The indicator $\bar{V}_{2 D}$ is the most suitable in this model.

Most of correlation coefficients between $\bar{S}_{3 D}$ and the OCIA are negative, and some of them are below the level of -0.4. However strength of the correlation coefficients is not stable. Therefore, it is difficult to use $\bar{S}_{3 D}$ as an air quality improvement indicator.

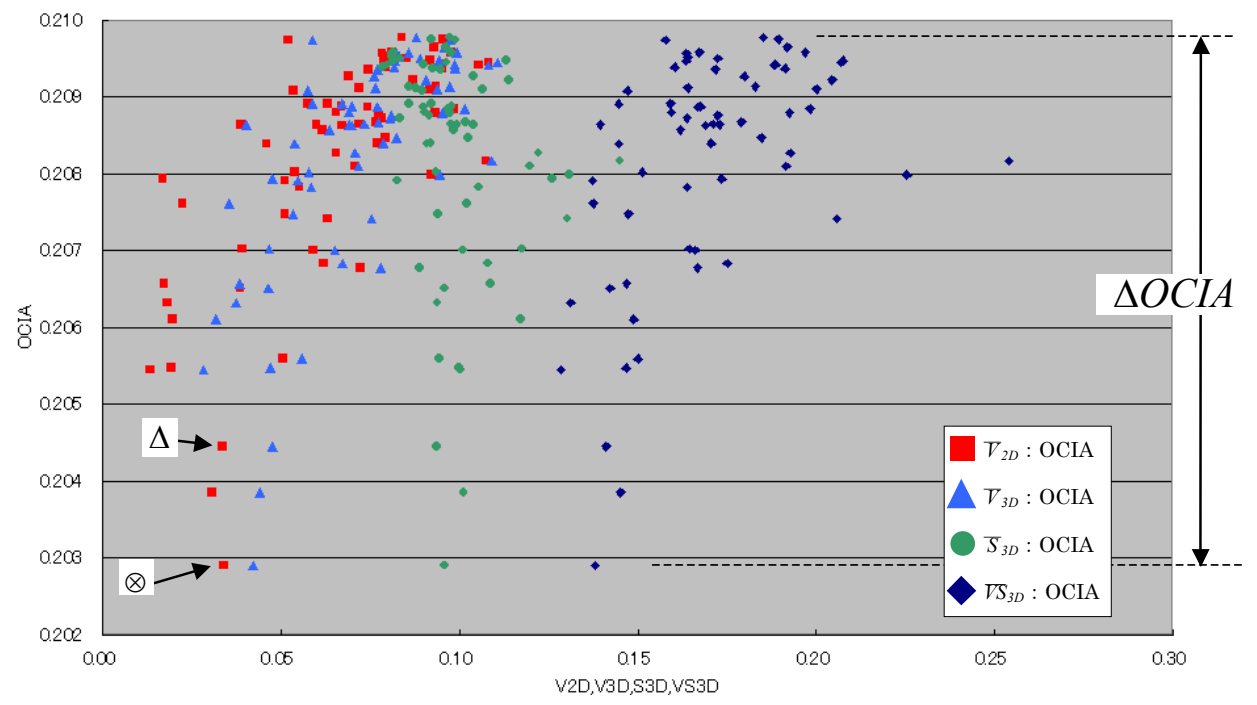

Fig.4 Correlations between indicators and the OCIA at No.2-body in Condition-A

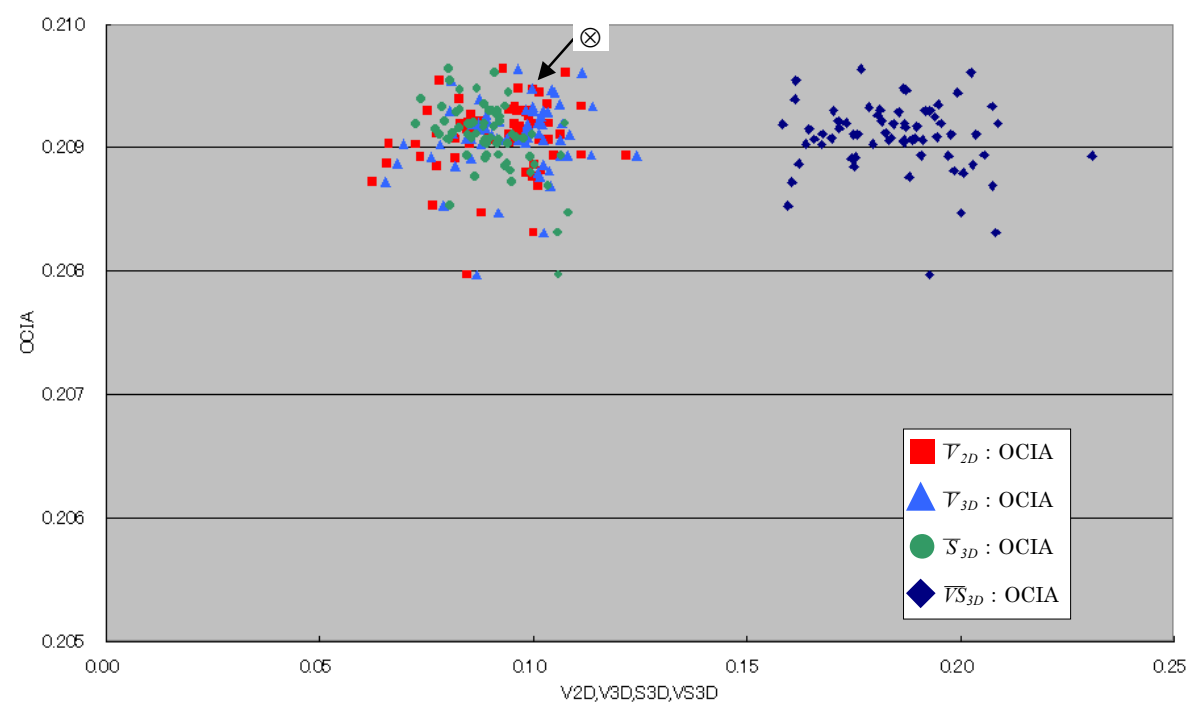

Fig.5 Correlations between indicators and the OCIA at No.4-body in Condition-A 


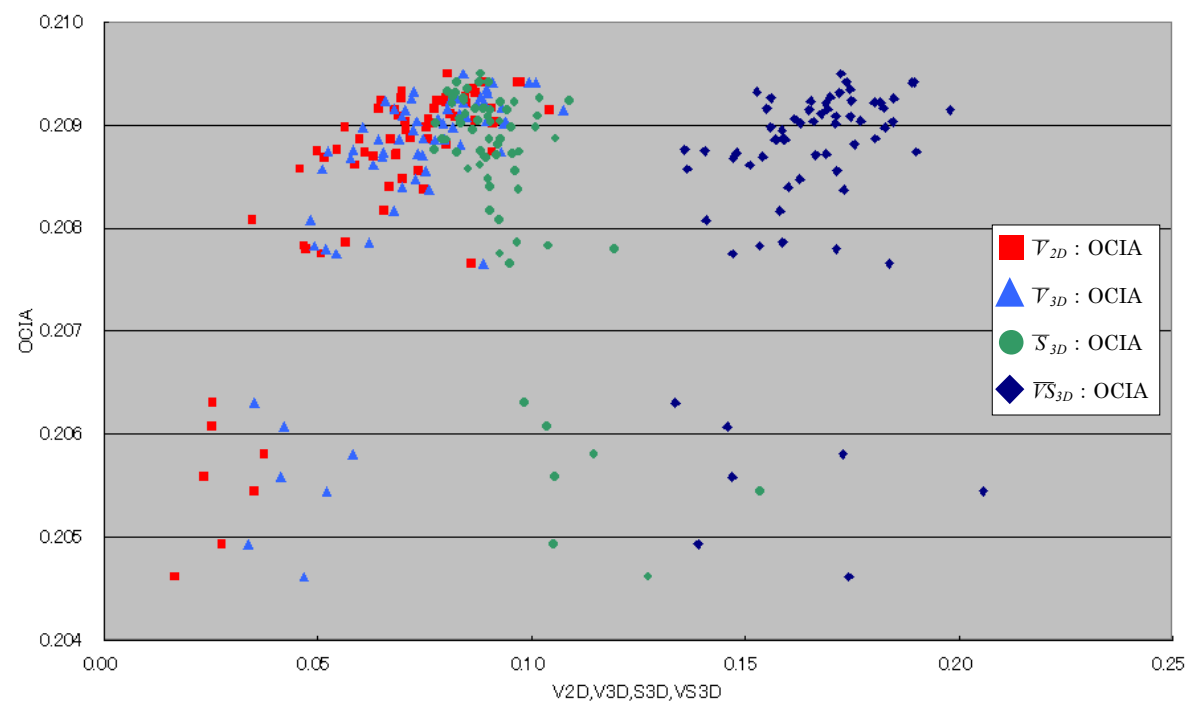

Fig.6 Correlations between indicators and the OCIA at No.2-body in Condition-B

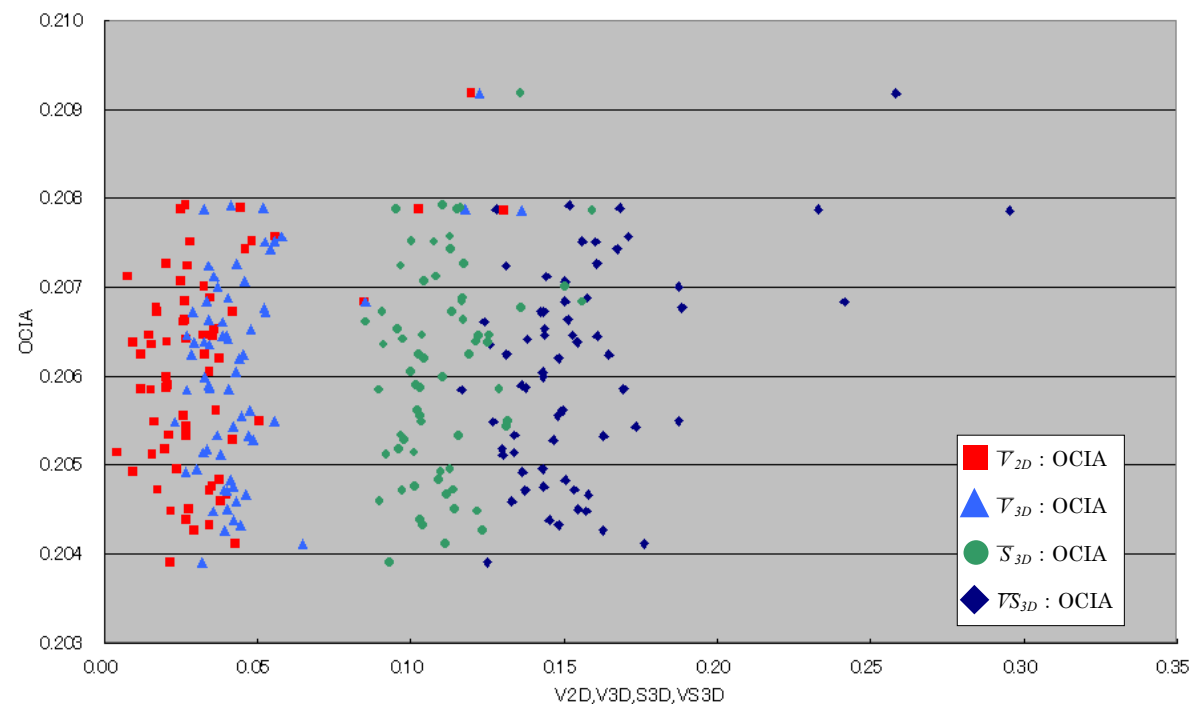

Fig.7 Correlations between indicators and the OCIA at No.2-body in Condition-D

\subsection{Observation of Flow State}

To find the mechanism related to the OCIA difference, two different examples to reduce renewal of air are shown in this section.

As one typical example of flow fields with low OCIA of No.2-body, a flow field of $x-y$ cross-section including the SIFM is shown in Fig.9. This chart indicates the flow field of time-averaged velocity during half of the inhalation period when the lowest value of the OCIA at No.2-body in condition-A is recorded. The correlation data corresponding to Fig.9 shows the point indicated by " $\otimes$ " mark in Fig. 4 (as the data of No.2-body) and Fig. 5 (as the data of No.4-body). From a close-up view near the mouth of No.2-body in Fig.9, magnitudes of velocity vectors at upper left part and lower right part in the SIFM of No.2-body are small, and a weak circular flow around mouth is also found. The flow state in these parts is almost regarded as stagnation. From the close-up view near the mouth of No.4-body in Fig.9, almost of the flow direction in the SIFM is upward, and suggests that a steady plume in front of the face of No.4-body exists. The plume enhances air renewal in the SIFM. 


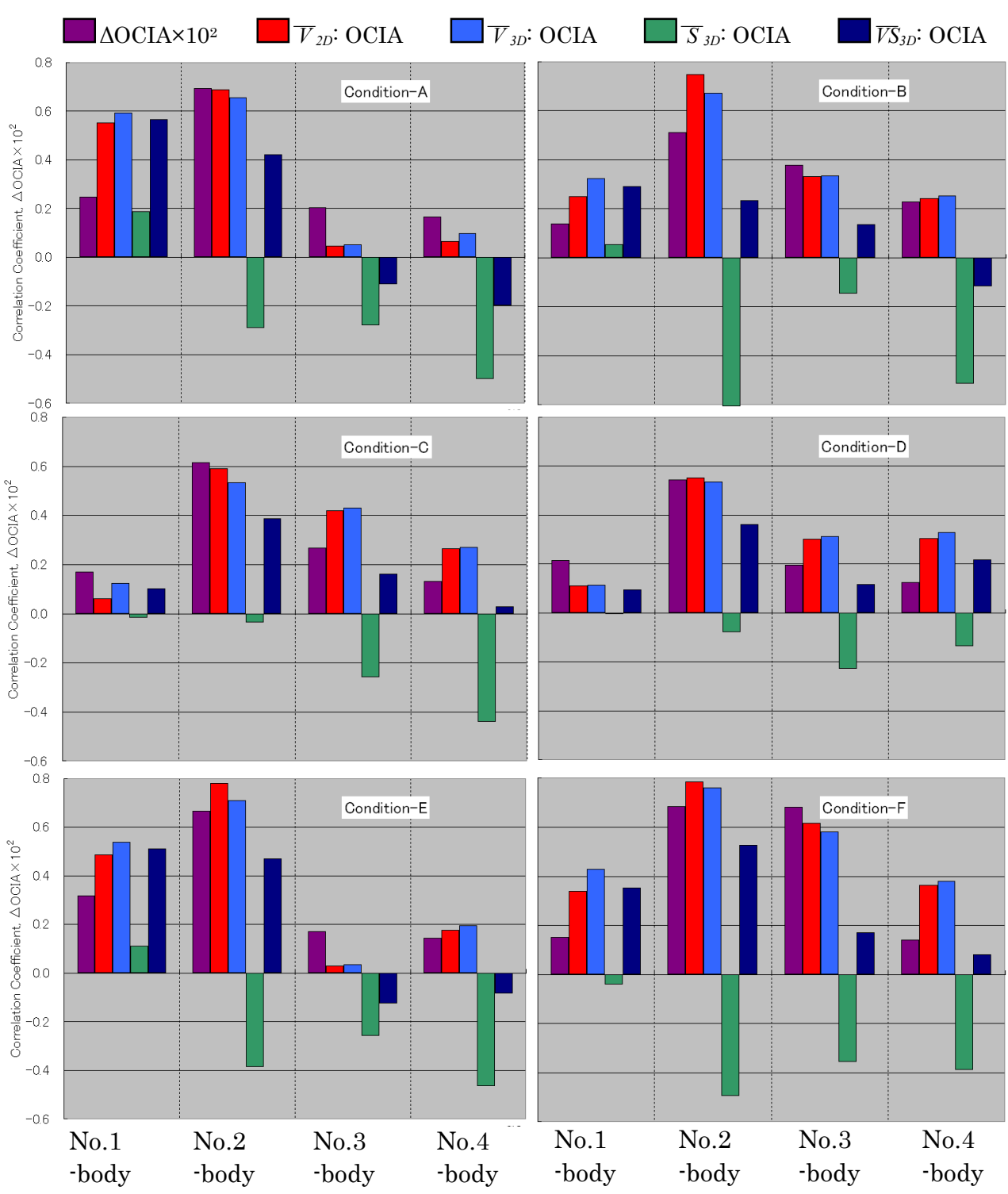

Fig.8 Correlation coefficients between the indicators and the OCIA

As the other typical example of flow fields with low OCIA of No.2-body, Fig.10 shows a flow field of $x-y$ cross-section including the SIFM. The correlation data corresponding to Fig.10 shows the point indicated by " $\Delta$ " mark in Fig. 4. Almost of the velocity vectors in the SIFM of No.2-body form circler flows just around the mouth of No.2-body. Since $\bar{V}_{2 D}$ (and also $\bar{V}_{3 D}$ ) is integration of time-averaged velocities all of the SIFM, in case of such circler flow, $\bar{V}_{2 D}$ (and $\bar{V}_{3 D}$ may) becomes zero. Due to the staying circular flow, the SIFM air is replaced little by outside fresh air.

From the above, $\bar{V}_{2 D}$ decreases in both the two cases, namely the stagnation and the circular flow just around mouth. Because flow in the z-direction due to inhalation may still remain, $\bar{V}_{3 D}$ becomes larger than $\bar{V}_{2 D}$ at the stagnation or the circular flow. Therefore, $\bar{V}_{2 D}$ effectively indicates the renewal state of SIFM air at each respiration cycle, even though either the stagnation or the circular flow causes inhibition of renewal.

Actually, to keep a steady stream through major part of the SIFM during a period of one inhaling action is effective in preventing deterioration of the OCIA. It is shown from observing velocity vectors in the $x-y$ cross-section including the SIFM of No.4-body. 

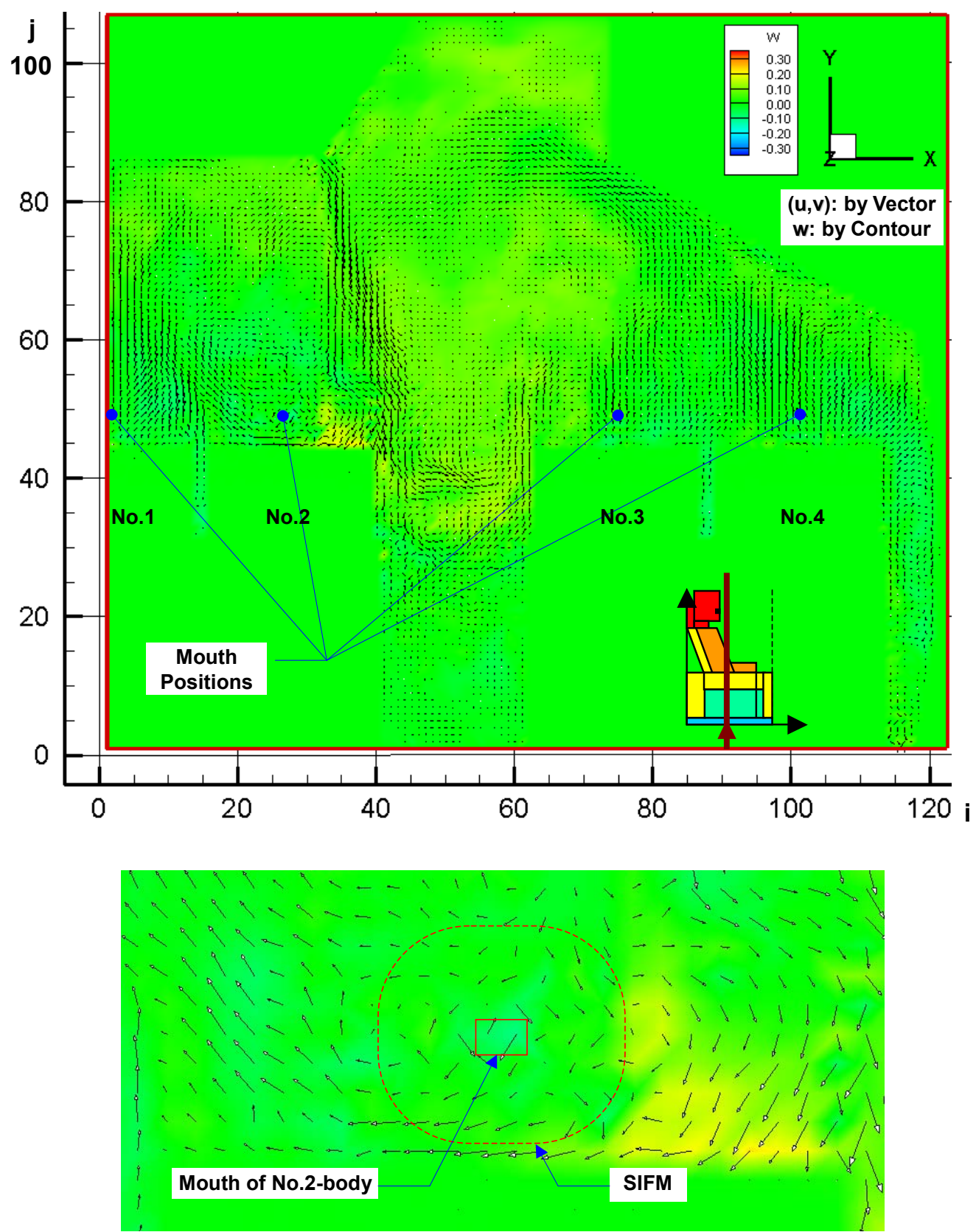

[Close-up-view 1] Flow field around the mouth of No.2-body

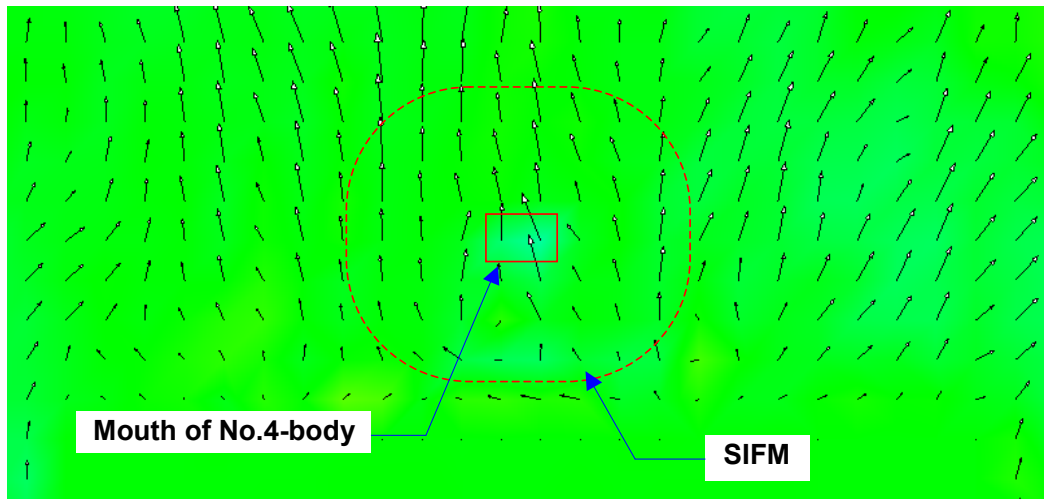

[Close-up-view 2] Flow field around the mouth of No.4-body

Fig.9 Flow field at the condition of " $\otimes$ " point in Fig. 4 and Fig. 5 


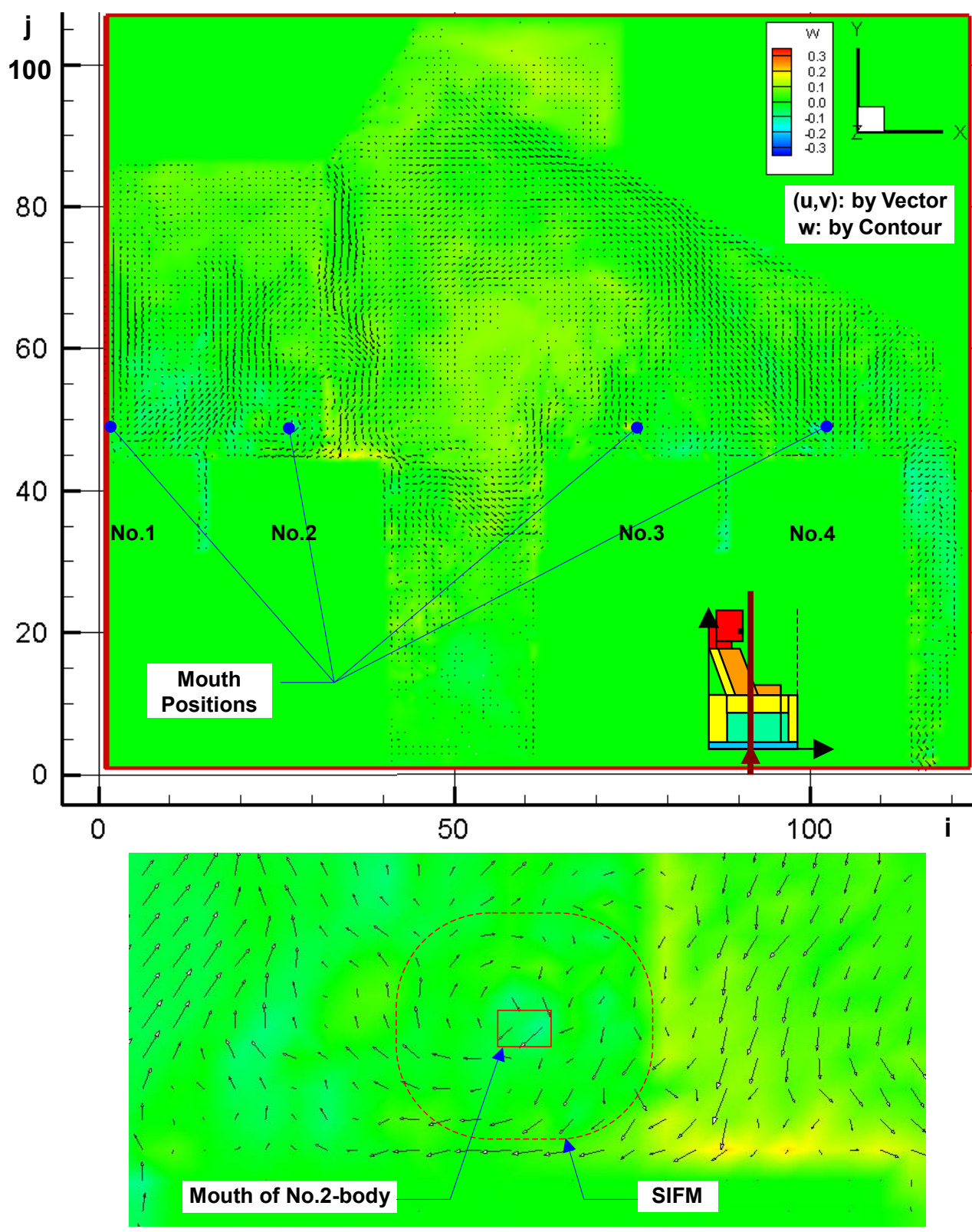

[Close-up-view 1] Flow field around the mouth of No.2-body

Fig.10 Flow field at the condition of " $\Delta$ " point in Fig. 4

From comparison with other flow field (not included in this paper), cause of the stagnation and the circular flow just around mouth is mainly a shift of tributary stream of supplied airflow generated by decline of supplied air temperature. Even though existing of such a shift, large scale circular flow along side ceiling still remains. It is the reason why steady plume is generated and the air quality is kept at No.3 body or No.4 body.

\section{Conclusions}

We applied the large-eddy simulation to the unsteady flow field in the model of aircraft cabin. The immersed-boundary method efficiently dealt with the complex configuration of the flow field. Simultaneously, the fields of temperature and oxygen concentration were simulated. Thus the influence of sinusoidal breathing of passengers could be taken into account in unsteady flow field by using LES. We selected an oxygen concentration of 
inhalation air (OCIA) in each respiration cycle as a representative of air quality. To calculate indicators for air quality, a space in front of mouth (SIFM) was used. The correlation between the OCIA and some possible indicators $\left[\bar{V}_{2 D}, \bar{V}_{3 D}, \bar{S}_{3 D}\right.$, and $\left.\overline{V S}_{3 D}\right]$ were evaluated. Major findings are as follows:

- The OCIA and indicators fluctuated in every inhaling action. The OCIA at particular position occasionally decreased under decreasing temperature of supplied air from ceiling.

- Intensity of time-averaged velocities $\left[\bar{V}_{3 D}\right]$ and that by cross-sectional components $\left[\bar{V}_{2 D}\right]$ correlated with the OCIA whenever the OCIA fluctuated widely (range of the OCIA [ $\triangle O C I A$ ] was greater than 0.005 , for example). The intensity of time-averaged $2 \mathrm{D}$ velocities $\left[\bar{V}_{2 D}\right]$ surpasses that of $3 \mathrm{D}$ velocities $\left[\bar{V}_{3 D}\right]$ in indication due to the simplicity.

- Intensity of velocity deviations $\left[\bar{S}_{3 D}\right]$ had negative correlation with the OCIA. However strength of the negative correlation was unstable. Intensity of velocity deviations $\left[\bar{S}_{3 D}\right]$ was inferior to $\bar{V}_{2 D}$ and $\bar{V}_{3 D}$ in indication. In addition, sum of $\bar{V}_{3 D}$ and $\bar{S}_{3 D}\left[\overline{V S}_{3 D}\right]$ was correlated weakly with the OCIA.

- Decreasing of the intensity of time-averaged velocities $\left[\bar{V}_{3 D}\right]$ (especially that by cross-sectional components $\left[\bar{V}_{2 D}\right]$ ) indicates occurrence of stagnation zone in the SIFM or growth of a circular flow around the mouth.

Inactivation of air renewal in the SIFM directly affects a deterioration of air quality and is exactly reflected in intensity of time-averaged velocities $\left[\bar{V}_{3 D}\right]$ (especially that by cross-sectional components $\left[\bar{V}_{2 D}\right]$ ). Hence the intensity of time-averaged 2D velocities $\left[\bar{V}_{2 D}\right]$ is an appropriate indicator of respiration air quality. Consequently, the indicator is useful to evaluate the effects of an air supply method such as personalized ventilation. Furthermore, the indicator related to the flow becomes a useful tool for optimization of air-conditioning flow design because the process includes computational effort for tremendous cases.

In an aircraft cabin, oxygen partial pressure decreases in cruising at high altitude since the cabin pressure approximates the atmospheric pressure at $2,438 \mathrm{~m}(8,000 \mathrm{ft})$ altitude for reduction of stresses at fuselage elements ${ }^{(22)}$. The decreased oxygen partial pressure is still in safety zone for an ordinary person. However, a serious health risk of some people with pulmonary disease was pointed out ${ }^{(1)}$. To solve this problem, we intend to utilize personalized ventilation. The indicator proposed in this paper will contribute toward making the personalized ventilation suitable for safety and comfort of passenger in cabin space.

\section{References}

(1) Committee on Air Quality in Passenger Cabins of Commercial Aircraft, Board on Environmental Studies and Toxicology, Division on Earth and Life Studies, National Research Council, "The Airliner Cabin Environment and the Health of Passengers and Crew", Washington D.C. National Academy Press, (2002), pp.1-72

(2) United States General Accounting Office, "AVIATION SAFETY, More Research Needed on the Effects of Air Quality on Airliner Cabin Occupants", GAO-04-54, (2004)

(3) American Conference of Governmental Industrial Hygienists (ACGIH), "2010 TLVs and BEIs with 7th Edition Documentation", (2010)

(4) Federal Regulations, Title 29 "Labor", Part1910 "Occupational Safety and Health Standards" http://ecfr.gpoaccess.gov/cgi/t/text/text-idx?c=ecfr\&tpl=\%2Findex.tpl

(5) ASHRAE Standard 62.1-2010, "Ventilation for Acceptable Indoor Air Quality", (2010) 
(6) Fanger, P. O., "Human Requirements in Future Air-Conditioned Environments", International Journal of Refrigeration, Vol.24, (2001), pp.148-153

(7) Melikov, A. K., Cermak, R., and Majer, M., "Personalized Ventilation: Evaluation of Different Air Terminal Devices", Energy and Buildings, Vol.34, (2002), pp.829-836

(8) Gao, N., and Niu, J., "CFD Study on Micro-Environment around Human Body and Personalized Ventilation”, Building and Environment, Vol.39, (2004), pp. 795-805

(9) Zitek, P., Vyhlidal, T., Simeunovic, G., Novakova, L., and Cizek, J., "Novel Personalized and Humidified Air Supply for Airliner Passengers", Building and Environment, Vol.45, (2010), pp.2345-2353

(10) Hiyama, K., Ishida, Y., and Kato S., "Building Simulation of Thermal Environment Using Response Factor Analysed by Three-Dimensional CFD”, Building Simulation, (2009), pp.474-480

(11)Zhu, S., Kato, S., Murakami, S., and Hayashi, T., "Study on Inhalation Region by Means of CFD Analysis and Experiment”, Building and Environment, Vol.40, (2005), pp.1329-1336

(12) Saito, H., Mitsuoka, D., Komai, T., Kawadu, T., Takao, K., Yamada, T., and Iwata, T., "Advanced System Program-Advanced Environmental Control System (Non-Bleed ECS)", Proceedings of 46th Aircraft Symposium, (2008), 3D4 (in Japanese)

(13) Saito, H., and Kajishima, T., "Thermal Field Analysis of Cabin Air using LES with Immersed Boundary Method", Proceedings of 2nd Asian Symposium on Computational Heat Transfer and Fluid Flow (ASCHT 09), (2009), C34

(14)Federal Regulations, Title 14 "Aeronautics and Space", Part 25, Section 25.831 "Ventilation" http://ecfr.gpoaccess.gov/cgi/t/text/text-idx?c=ecfr\&tpl=\%2Findex.tpl

(15) Boeing Commercial Airplanes Home Pages http://www.boeing.com/commercial/index.html

(16)Zhu, S., Hayashi, T., Kato, S., and Murakami, S., "Investigation of Flow Field in Human's Respiration Area in a Calm Environment by Visualization Experiment and Numerical Analysis", AIJ Journal of Environmental Engineering, No.583 (2004), pp.37-42 (in Japanese)

(17) Kajishima, T., Takiguchi, S., Hamasaki, H., and Miyake, Y., "Turbulence Structure of Particle-Laden Flow in a Vertical Plane Channel Due to Vortex Shedding", JSME International Journal, Series B, Vol.44, No.4 (2001), pp.526-535

(18) Mittal, R., and Iaccarino, G., "Immersed Boundary Methods", Annual Review Fluid Mechanics, Vol.37, (2005), pp.239-261

(19) Murata, F., Hirohata, M., Taniguchi, N., and Kobayashi, T., "Flow Control of Coaxial Mixing Jet by Vortex Excitation and Prediction of Vortex Excitation Frequencies", SEISAN-KENKYU Vol. 53, No. 1, (2001), pp.53-56 (in Japanese)

(20) Eidson, T. M., "Numerical simulation of turbulent Rayleigh-Benard convection using subgrid scale modeling”, Journal of Fluid Mechanic, Vol.158, (1985), pp.245-268

(21) Hiroshige, T., and Kato, M., "Elementary Physiology", Nanzando Co. Ltd., (1992), pp.89-106 (in Japanese)

(22)Federal Regulations, Title 14 "Aeronautics and Space", Part 25, Section 25.841 "Pressurized cabins"

http://ecfr.gpoaccess.gov/cgi/t/text/text-idx?c=ecfr\&tpl=\%2Findex.tpl 